\section{Nichttuberkulöse Mykobakteriose der Lungen durch Mycobacterium smegmatis}

J. Schreiber ${ }^{1}$, U. Burkhardt ${ }^{1}$, S. Rüsch-Gerdes ${ }^{2}$, M. Amthor ${ }^{3}$, E. Richter ${ }^{2}$, M. Zugehör ${ }^{1}$, W. Rosahl', M. Ernst ${ }^{2}$

${ }^{1}$ Städtisches Klinikum Dessau, Akademisches Lehrkrankenhaus der Martin-Luther-Universität Halle - Wittenberg, Klinik für Innere Medizin

2 Zentrum für Medizin und Biowissenschaften Borstel, Nationales Referenzzentrum für Mykobakterien

${ }^{3}$ Diakoniekrankenhauses Rothenburg/W., Institut für Pathologie

blood mononuclear cells. Treatment with Rifabutin, Ethambutol, Clarithromycin and Ofloxacin resulted in complete clinical and roentgenological resolution.

terium smegmatis ist eine Rarität. Ein 51-jähriger männlicher, HIV-negativer Patient ohne bronchopulmonale Vorerkrankungen, jedoch mit einem Zustand nach Gastrektomie und Splenektomie, erkrankte mit Husten, Gewichtsverlust und Nachtschweiß. Röntgenologisch und computertomographisch waren ausgedehnte beidseitige fleckförmige, teilweise konfluierende Infiltrate nachweisbar. Histologisch fand sich in den transbronchialen Bioptaten das Bild einer chronisch-karnifizierenden Pneumonie und in einer perthorakalen Feinnadelpunktion verkäsende epitheloidzellige Granulome und säurefeste Stäbchen, die mittels PCR und anschließender Sequenzierung als $M$. smegmatis identifiziert wurden. Im Sputum und Bronchialsekret kein mikroskopischer Nachweis säurefester Stäbchen, aber wiederholt Wachstum von M. smegmatis. In allen Untersuchungsmaterialien kein Nachweis von Tuberkulosebakterien (Nukleinsäureamplifikationstechnik [NAT], Kultur). Immunologische Untersuchungen zeigten eine Reduktion der CD4+Lymphozyten und eine erniedrigte Interferon $\alpha$ - und $-\gamma$-Produktion der mononukleären Zellen des peripheren Bluts. Unter einer Therapie mit Rifabutin, Ethambutol, Clarithromycin und Ofloxacin kam es zu einer vollständigen röntgenologischen und klinischen Regredienz.

Nontuberculous Mycobacteriosis due to Mycobacterium smegmatis: Nontuberculous mycobacteriosis due to $\mathrm{M}$. smegmatis is a rarity. We report on the case of a 51 year old male HIV-seronegative patient whitout predisposing bronchopulmonary disease, but with a state after gastrectomy and splenectomy who developed unproductive cough, night sweat and weight loss. The chest radiograph and thoracic CT showed wide-spread bilateral patchy infiltrations. Histological examination of transbronchial biopsies revealed chronic carnificating pneumonia. A perthoracic fine-needle biopsy showed caseating epitheloid cell granulomas with acid fast bacilli. These were identified as $\mathrm{M}$. smegmatis by PCR with subsequent sequencing. Acid fast bacilli could not be detected microscopically neither in sputum nor in bronchial secretions, however M. smegmatis has been repeatedly detected by culture in these materials. In neither material tubercle bacilli have been detected by nucleic acid amplification (NAT) or culture. Immunologic investigations revealed a reduced number of CD4+ lymphocytes and a reduction of interferon $\alpha$ - and $-\gamma$-synthesis by peripheral

Pneumologie 2001; 55: 238-243

(c) Georg Thieme Verlag Stuttgart · New York ISSN 0934-8387

\section{Klinischer Untersuchungsbefund}

Reduzierter Allgemein- und Ernährungszustand, verschärftes Atemgeräusch über beiden Lungen, Herzaktion tachykard, Töne rein, Abdomen weich mit reizlosen Narbenverhältnissen.

Labor

BSG 74/90 mm n.W., Ery 5,2/pl, Hb 11,7 mmol/1, MCV 77,7 fl, MCH 24 pg, MCHC 32,2 g/dl, Leuko 13,8/nl, Thrombo 859/nl, 
CrP 124,2 mg/l, $\gamma$ GT 2,16 U/l, AP 12,24 U/l, LDH 8,1 U/l, Elektrolyte, Kreatinin, Harnstoff, Harnsäure, GOT, GPT, Bilirubin, Amylase, Lipase, Immunglobuline, TSH, Urinstatus ohne pathologischen Befund, HIV1/2 negativ, Blutgasanalyse: im Normbereich.

EKG: unauffällig

Röntgenuntersuchung der Thoraxorgane (Abb.1): In beiden Lungen Nachweis von ausgedehnten fleckförmigen, teilweise konfluierenden Infiltrationen.

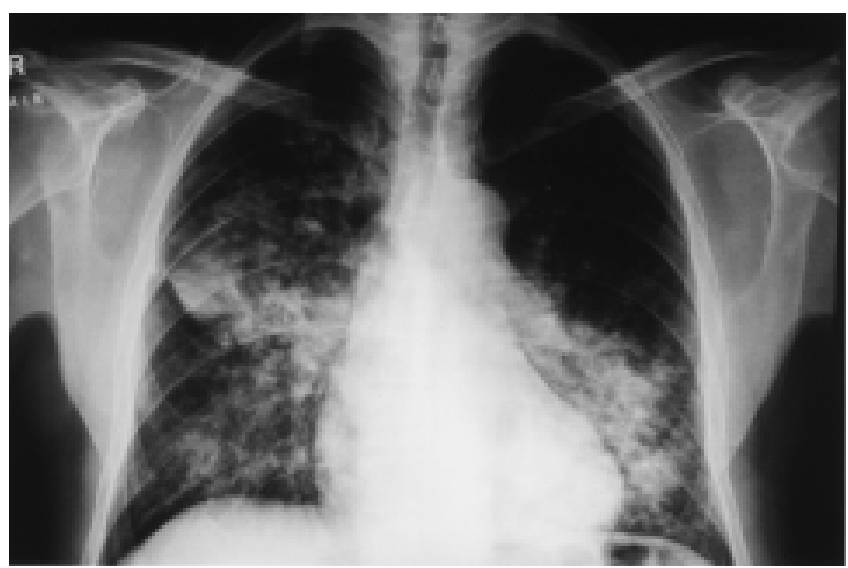

Abb. 1 Ausgedehnte Infiltrationen in beiden Lungen.

Computertomographie der Thoraxorgane (Abb. 2): Im Lungenparenchym beidseits ausgedehnte konfluierende, teilweise rundherdartig imponierende Infiltrationen ohne Hohlraumbildung vorwiegend in den dorsalen und basalen Segmenten, keine pathologisch vergrößerten mediastinalen Lymphknoten.

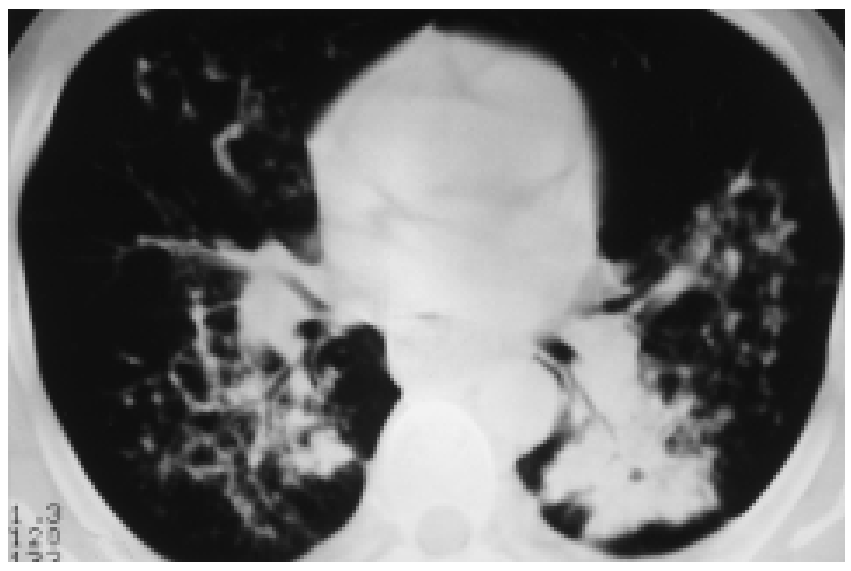

Abb. 2 Computertomographie der Thoraxorgane mit ausgedehnten Infiltrationen.

Lungenfunktionsdiagnostik (Bodyplethysmographie, Diffusionsmessung): Normalbefund, keine Obstruktion, keine Restriktion, keine Überblähung, Diffusionskapazität nicht eingeschränkt.
Tuberkulintest (10 TE i.c.): $7 \mathrm{~mm}$.

Gastroskopie: Z.n. totaler Gastrektomie, Anastomose und Ösophagus unauffällig.

Bronchoskopie: geringe Zeichen einer chronischen Bronchitis, kein Tumornachweis.

Bronchoalveoläre Lavage: 61\% Makrophagen (ohne Lipideinschlüsse), $17 \%$ neutrophile Granulozyten und $22 \%$ Lymphozyten $(\mathrm{CD} 4 / \mathrm{CD} 8=2,28)$.

Histologie von transbronchialen Lungenbioptaten: (Abb. 3,4): Nachweis einer organisierenden, noch fibrinreichen Pneumonie ohne Anhalt für Malignität, kein Nachweis von Granulomen, kein Nachweis von säurefesten Stäbchen.

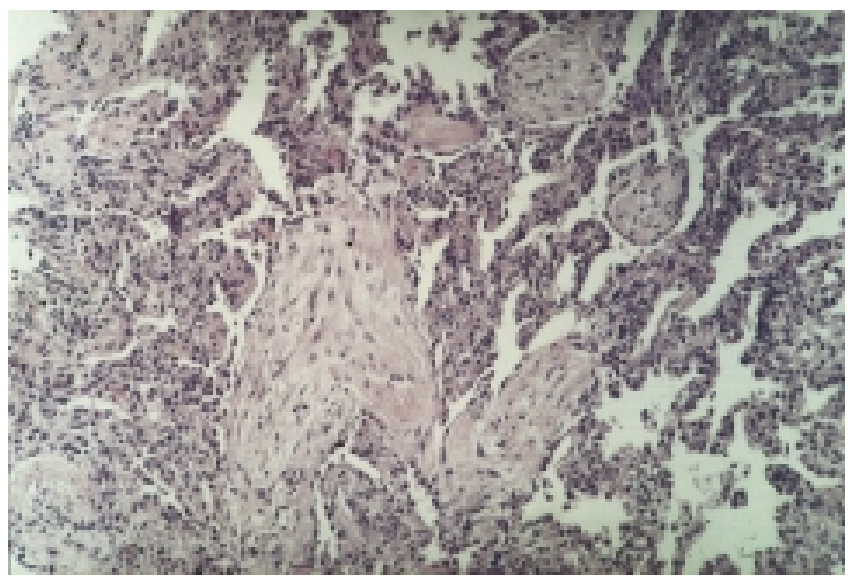

Abb. 3 Karnifizierende Pneumonie in einer transbronchialen Lungenbiopsie (HE-Färbung).

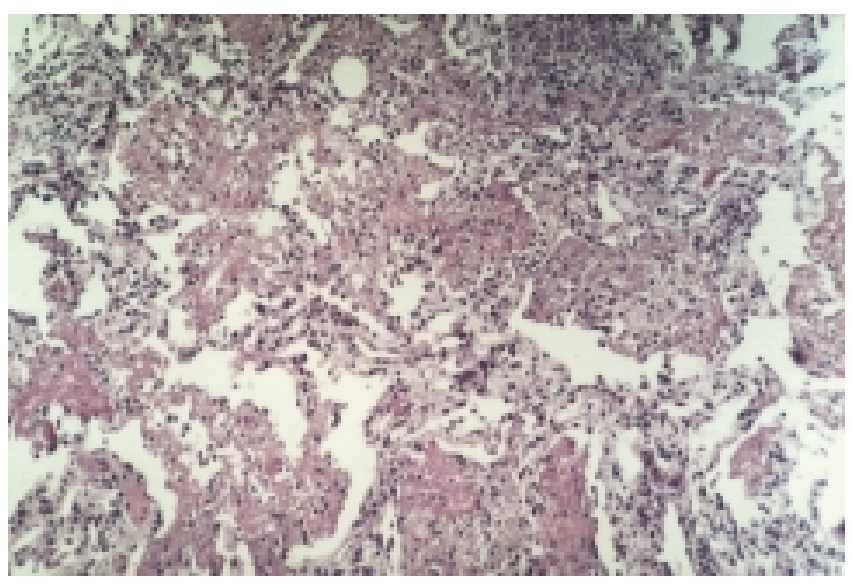

Abb. 4 Organisierende, fibrinreiche Pneumonie in einer transbronchialen Lungenbiopsie (HE-Färbung).

Bakteriologie: Im Sputum, im Bronchialsekret und in der BAL kein Keimnachweis, kein Nachweis säurefester Stäbchen, NAT auf M. tuberculosis und Kulturen auf Mykobakterien negativ.

Verlauf: Auf Grundlage der o.g. Untersuchungen Einleitung einer erneuten unspezifischen Antibiotikatherapie und einer systemischen Glukokortikosteroidtherapie mit 40 mg Predni- 
solon/die. Unter dieser Therapie subjektiv zunächst Besserung der Beschwerdesymptomatik und röntgenologisch geringe Regredienz der Infiltrationen. Im weiteren Verlauf jedoch subjektive Verschlechterung und röntgenologisch und computertomografisch Progredienz der Befunde mit Ausbildung von multiplen pulmonalen Rundherden. Deshalb erneute Diagnostik:

Bronchoskopie: unveränderter makroskopischer Befund.

Histologie von transbronchialen Lungenbioptaten: alveoläres Lungenparenchym mit intraalveolären Granulationspfröpfen, schüttere lympho-granulozytäre Infiltrate, Fibroblasten, in einigen Abschnitten zeigt das Granulationsgewebe auch Ansammlungen von Epitheloidzellen, welche zum Teil rosettenartig angeordnet sind, zentral mit vermehrten neutrophilen Granulozyten, keine mehrkernigen Riesenzellen, keine typischen käsigen Nekrosen. Beurteilung: Lungenparenchym mit einzelnen Epitheloidzellgranulomen und mit einzelnen kleinen Herden einer organisierenden Pneumonie, kein Nachweis von säurefesten Stäbchen, kein Anhalt für Malignität.

Histologie einer perthorakalen Feinnadelbiopsie: (Abb. 5, 6): Nachweis verkäsender epitheloidzelliger Granulome und zahlreicher säurefester Stäbchen.

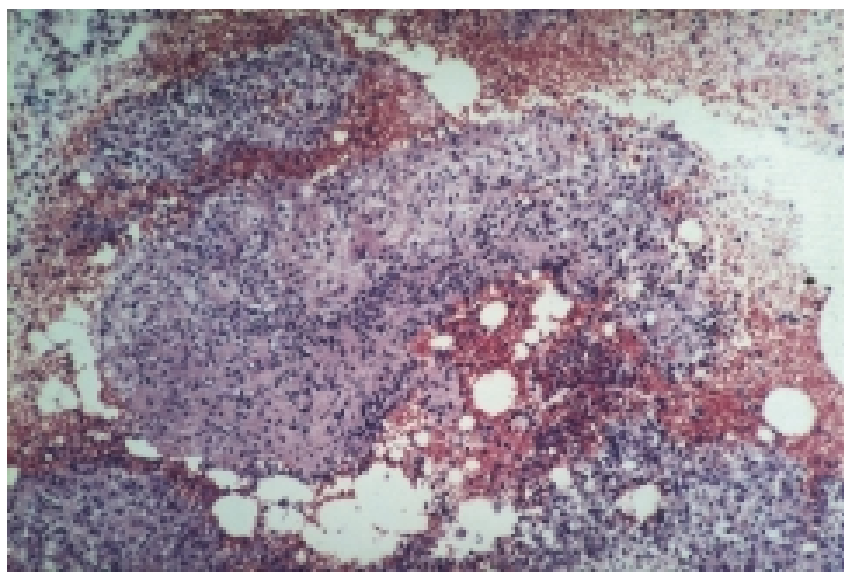

Abb. 5 Epitheloidzellige Granulome in einer perthorakalen Feinnadelbiopsie (HE-Färbung).

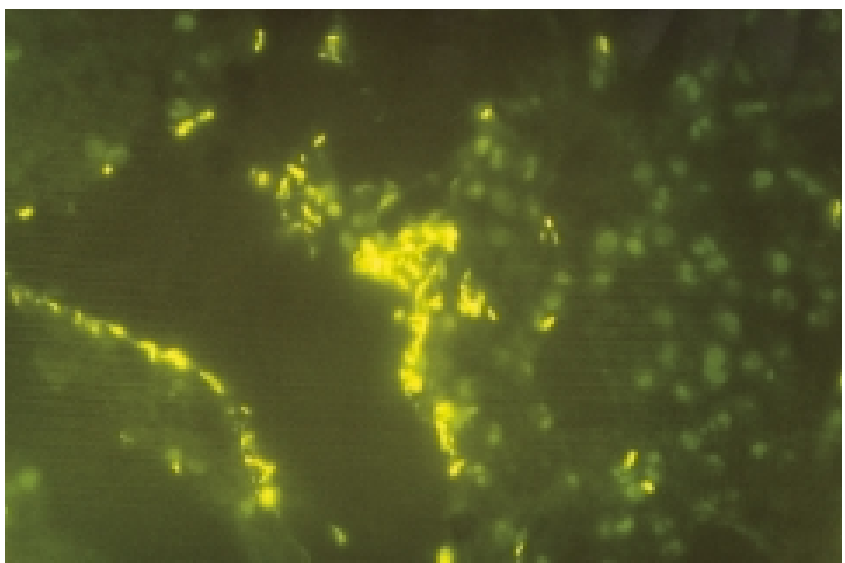

Abb. 6 Massenhaft säurefeste Stäbchen in einer perthorakalen Feinnadelbiopsie (Rhodamin-Auramin-Färbung).
Bakteriologie: Im Sputum und im Bronchialsekret kein mikroskopischer Nachweis säurefester Stäbchen, NAT auf M. tuberculosis negativ. Im perthorakalen Feinnadelaspirat Nachweis von M. smegmatis durch PCR und anschließende Sequenzierung. Im weiteren Verlauf kultureller Nachweis von $M$. smegmatis im Sputum und im Bronchialsekret.

Resistenztestung: sensibel gegen Ethambutol, Streptomycin, Ofloxacin, Capreomycin, intermediär sensibel gegen Rifabutin, resistent gegen Isoniazid, Rifampicin, Pyrazinamid, Cycloserin, Protionamid und Clarithromycin.

Therapie und weiterer Verlauf: Nach Vorliegen der o.g. histologischen Ergebnisse Einleitung einer tuberkulostatischen 4fach-Therapie mit Isoniazid, Rifampicin, Pyrazinamid und Ethambutol. Nach Erregertypisierung Fortsetzung der Therapie mit Rifabutin, Ethambutol, Clarithromycin und Ofloxacin. Unmittelbar nach Therapiebeginn kam es zur Ausbildung eines ausgedehnten linksseitigen Pleuraergusses (lymphozytenreiches Exudat ohne Erregernachweis), der die Anlage einer Thoraxdrainage erforderlich machte. Nach einer insgesamt einjährigen medikamentösen Therapie vollständige Normalisierung der Beschwerdesymptomatik und der klinischen, laborchemischen und röntgenologischen Befunde.

In Kenntnis der Diagnose und unter antimykobakterieller Therapie wurden folgende immunologische Untersuchungen durchgeführt:

\section{Immunfluoreszenz an mononukleären Zellen (MNZ) des peripheren Bluts}

CD3 (reife T-Ly.): 34\% (NB: $60-90 \%)$,

CD4 (T-Helfer-Ly.): 15\% (NB: 35-60\%),

CD45RA/CD4 (Suppressor-Inducer Ly., naive Helfer T-Ly.): 7\% (NB: $15-30 \%$,

CD45R0/CD4 (Memory T-Helfer Ly): 5\% (NB: 15-30\%),

CD38 (unreife T-Ly, aktivierte T-Ly und B-Ly): 52\% (NB: 4$20 \%)$,

Verhältnis CD4/CD8 : 0,44 (NB: 1,2-2,7),

Monozytenanteil in den MNZ: 28\% (NB: 10-20\%).

Im Normbereich waren CD2 (reife + unreife T-Ly, einige NKZellen), CD8 (zytotoxische T-Ly, Suppressor T-Ly), CD25 (IL-2Rezeptor, aktivierte T-Lymphozyten, Monozyten), CD14 (Monozyten).

\section{In vitro stimulierte Zytokinfreisetzung in Zellkulturen}

Die Untersuchungen wurden mit verdünntem Vollblut und an isolierten mononukleären Zellen (MNZ) des peripheren Bluts durchgeführt.

Reduktion der NDV-(Newcastle Disease Virus) induzierten Interferon $\alpha$ (IFN $\alpha$ )-Freisetzung im peripheren Blut auf $91 \mathrm{pg}$ / $\mathrm{ml}(\mathrm{NB}: 300-1500 \mathrm{pg} / \mathrm{ml})$ und aus MNZ auf $138 \mathrm{pg} / \mathrm{ml}$ (NB: $500-2500 \mathrm{pg} / \mathrm{ml})$.

Reduktion der PHA-(Phytohämagglutinin) induzierten Interferon $\gamma($ IFN $\gamma$ )-Freisetzung im peripheren Blut auf $2902 \mathrm{pg} / \mathrm{ml}$ und der anti-CD3 induzierten IFN $\gamma$-Freisetzung aus MNZ auf $890 \mathrm{pg} / \mathrm{ml}$ (NB jeweils: $8000-50000$ pg/ml). 
Die PHA und anti-CD3-induzierte tumor-necrosis-factor $\alpha$ (TNF $\alpha$ )-Freisetzung aus dem peripheren Blut und aus MNZ war im Normbereich.

\section{IFN $\gamma$-Freisetzung aus MNZ nach Stimulation mit dem M. smegmatis-Stamm des Patienten}

MNZ wurden mit aufsteigenden Keimzahlen des patienteneigenen $\mathrm{M}$. smegmatis-Stammes (0,05 bis 2 Mio./0,2 Mio. PBMC) und den Zytokinen IL12 und IL15 und mit anti-IL-10 cokultiviert und die IFN $\gamma$-Freisetzung im Überstand gemessen. Es war ein nahezu vollständiges Fehlen einer adäquaten IFN $\gamma$-Synthese auf M. smegmatis allein und bei Cokultivierung mit IL12 und anti-IL10 nachweisbar. Kontrollpersonen zeigten positive Reaktionen.

\section{Phagozytenfunktionstests an MNZ und isolierten Granulozyten (PMN) des peripheren Bluts}

Die stimulusinduzierte Freisetzung von Superoxid und reaktiven Folgeprodukten aus Phagozyten wurde mit Hilfe der Chemilumineszenz-(CL)-Messung bestimmt und war für MNZ unauffällig. In PMN zeigte sich für eine Stimulation mit Kaninchen-IgG beladenen Schafserythrozyten eine Reduktion der CL-Aktivität auf 2398 Counts $\times 10^{-3} / 20$ min (NB: $4000-$ 20000 Counts $\times 10^{-3} / 20 \mathrm{~min}$ ). Die CL-Aktivitäten nach Stimulation mit Zymosan, Latexpartikeln, FMLP und mit Kaninchen-IgG beladenen Schafserythrozyten $+5 \%$ aktivierbarem Serum waren im Normbereich.

\section{Diskussion}

Die NTM wurden schon wenige Jahre nach den Tb-Bakterien, Mycobacterium smegmatis bereits 1884 entdeckt. Es verdankt seinen Namen dem erstmaligen Nachweis in syphilitischen Ulzera und genitalen Sekreten, ist jedoch ein ubiquitärer Umweltkeim, der vorwiegend im Wasser vorkommt. $M$. smegmatis gehört $\mathrm{u}$.a. mit M. fortuitum und $\mathrm{M}$. chelonae $\mathrm{zu}$ den schnell wachsenden Mykobakterien ( Runyon Gruppe IV) [5].

Humane Erkrankungen durch M. smegmatis sind sehr selten und betreffen vorwiegend die Haut und Weichteile, meist als Wundinfektionen $[4,6]$. Pulmonale Infektionen, Endokarditiden und Lymphadenitiden sind Raritäten $[4,7]$. Eine disseminierte M. smegmatis-Infektion wurde bisher erst einmal, bei einem Kind mit einem angeborenen Interferon $\gamma$-Rezeptordefekt beschrieben [8]. In einer Analyse von 22 Patienten mit einer M. smegmatis-Infektion waren 19 von Haut- und Weichteilinfektionen betroffen, wobei eine Häufung von Wundinfektionen nach kardiochirurgischen Eingriffen auffiel. Bei zwei Patienten erfolgte der M. smegmatis-Nachweis im Sputum, wobei keine Angaben zur klinischen Relevanz gemacht wurden. Lediglich bei einem Patienten mit einer Lipoidpneumonie konnte die Relevanz der M. smegmatisInfektion durch einen Bakteriennachweis aus einem Lungenbioptat erbracht werden [4]. Lipoidpneumonien scheinen ein prädisponierender Faktor für eine M. smegmatis-Infektion zu sein. Bereits in der ersten Beschreibung einer pulmonalen $\mathrm{M}$. smegmatis-Infektion wurde über einen Patienten berichtet, bei dem wiederholte Ölinstillationen in sein Tracheostoma erfolgt sind [9]. Es wird vermutet, dass die Erreger in einer fettreichen Umgebung gegen Enzyme geschützt sind und dass
Fette einen wachstumsstimulierenden Effekt haben $[10,11]$ Dies wird auch als eine Ursache für die seit langem bekannte Assoziation zwischen Ösophaguserkrankungen wie der Achalasie und dem Auftreten von nichttuberkulösen Mykobakteriosen betrachtet $[4,10-15]$. Es wird vermutet, dass die Achalasie mit einem fettreichen Milieu im Ösophagus den schnellwachsenden Mykobakterien günstige Vermehrungsbedingungen bietet und dass es zusätzlich zu rezidivierenden Mikroaspirationen von Nahrungsfetten kommt. Bei unserem Patienten lag ein Zustand nach Gastrektomie und Splenektomie vor. Auch wenn sich keine Anhaltspunkte für eine Refluxsymptomatik oder rezidivierende Aspirationen von Nahrungsfett fanden, ist zu vermuten, dass dies einen prädisponierenden Faktor für die Mykobakteriose dargestellt hat.

Weitere Erkrankungen, die das Auftreten von nichttuberkulösen Mykobakteriosen begünstigen können, sind pulmonale Vorschädigungen, z. B. Pneumokoniosen, posttuberkulöse Veränderungen, chronische Bronchitis oder Bronchiektasen, eine immunsuppressive Therapie oder systemische resistenzmindernde Erkrankungen wie Diabetes mellitus, Kollagenosen oder eine HIV-Infektion [16-18]. Derartige prädisponierende Faktoren lagen bei unserem Patienten nicht vor. Unabhängig von lokalen resistenzmindernden Erkrankungen wurden bei Patienten mit MOTT immunologische Veränderungen beschrieben, die eine Infektion mit den fakultativ pathogenen Keimen begünstigen. So konnte gezeigt werden, dass bei diesen Patienten die IFN $\gamma$ - und TNF $\alpha$-Produktion der mononukleären Zellen des peripheren Blutes signifikant erniedrigt ist [19]. IFN $\gamma$ ist für die Aktivierung von Makrophagen und in der Abwehr von Mykobakterien entscheidend [20,21]. TNF $\alpha$ ist essenziell in der Granulombildung [22] und IFN $\alpha$ moduliert die zelluläre Immunität in Richtung einer Th1-Antwort [23]. Auch bei unserem Patienten zeigte sich die erniedrigte IFN $\gamma$ - und IFN $\alpha$-Synthese sowohl auf PHA und anti-CD3 als auch auf seinen eigenen Mykobakterienstamm. Die TNF $\alpha-$ Produktion hingegen war normal. Es ist wahrscheinlich, dass die immunologischen Defizite einen prädisponierenden Faktor für die Infektion dargestellt haben.

Weiterhin waren bei unserem Patienten eine Erniedrigung der CD4+-Lymphozyten und des CD4/CD8-Quotienten im peripheren Blut nachweisbar. Diese Veränderungen sind nicht typisch für Patienten mit MOTT [19], können jedoch Folge der Splenektomie sein $[24,25]$. Da bei Patienten nach Splenektomie eine normale Interferon-Produktion beschrieben wurde, ist zu vermuten, dass die reduzierte IFN $\gamma$ - und IFN $\alpha$-Produktion einen unabhängigen zusätzlichen prädisponierenden Faktor darstellte. Für diese Annahme spricht auch, dass eine Häufung von MOTT bei splenektomierten Patienten bisher nicht beschrieben wurde.

Das klinische und röntgenmorphologische Bild von nichttuberkulösen Mykobakteriosen ist der Tuberkulose sehr ähnlich. Auch das gemeinsame histologische Substrat - die verkäsende epitheloidzellige Granulomatose - gestattet keine morphologische Differenzialdiagnose [17]. Bemerkenswert erscheint uns bei unserem Patienten, dass in mehreren und wiederholten transbronchialen Biopsien das histologische Bild einer organisierenden Pneumonie vorlag und erst eine perthorakale Punktion die typischen histologischen Veränderungen erbrachte. Der Krankheitsverlauf weist jedoch darauf 
hin, dass beide histologischen Veränderungen Ausdruck der Mykobakteriose waren.

Die Diagnose nichttuberkulöser Mykobakteriosen wird dadurch erschwert, dass beim Nachweis von MOTT immer eine Infektion von einer Kolonisation oder Kontamination unterschieden werden muss. Deshalb wird ein mehrfacher kultureller Nachweis der gleichen MOTT-Spezies gefordert. In den 1990 publizierten und 1997 aktualisierten Empfehlungen der ATS (American Thoracic Society) zur Diagnostik und Therapie von Krankheiten durch nichttuberkulöse Mykobakterien [27] wurden bakteriologische Diagnosekriterien definiert. Bei unserem Patienten war M. smegmatis wiederholt im Sputum und im Bronchialsekret und einmalig in einem steril gewonnenen Lungenbioptat bei histologischem Nachweis von epitheloidzelligen Granulomen nachweisbar, so dass die Relevanz dieses Erregers als gesichert betrachtet werden kann. $\mathrm{Zu}$ keinem Zeitpunkt konnten kulturell oder mittels NAT Tuberkulosebakterien nachgewiesen werden.

Die röntgenologischen Veränderungen der Lungen bei MOTT sind vielgestaltig. Während die „klassische Infektion“ röntgenologisch von einer Tuberkulose nicht zu unterscheiden ist, liegen bei der „nichtklassischen Form“ zentrilobuläre Rundherde besonders in der Lingula und im Mittellappen und Bronchiektasen vor. Besonders bei Patienten mit einem erworbenen Immundefekt sind mediastinale und hiläre Lymphknotenschwellungen häufig. Rundherde können auch bei asymptomatischen Patienten auftreten [28,29]. Bei unserem Patienten lagen ausgedehnte Infiltrationen im Lungenparenchym ohne Hohlraumbildungen und ohne Lymphknotenschwellungen vor. Rundherdartige Strukturen entwickelten sich im Krankheitsverlauf. Das Auftreten eines Pleuraergusses bei einer M. smegmatis-Infektion wurde in der recherchierten Literatur nicht beschrieben.

Die Therapie von nichttuberkulösen Mykobakteriosen ist oft langwierig und schwierig [30-32]. Die publizierten Fälle einer $M$. smegmatis-Infektion zeigten jedoch alle einen günstigen Verlauf unter medikamentöser Therapie. Auch bei unserem Patienten kam es durch eine Behandlung mit Rifabutin, Ethambutol, Clarithromycin und Ofloxacin zu einer vollständigen Regredienz der Beschwerden und der klinischen, laborchemischen und röntgenologischen Befunde.

\section{Literatur}

${ }^{1}$ Schönfeld N, Matthiessen W. Nichttuberkulöse Mykobakteriosen. In: Konietzko N, Loddenkemper R (Hrsg.). Tuberkulose. Stuttgart: Georg Thieme Verlag, 1999: 235-246

2 Nightingale SD, Byrd LT, Southern PM, Jokusch JD, Cal SX, Wynne BA. Incidence of Mycobacterium avium-intracellulare complex in human immunodeficiency virus-positive patients. J Infect Dis 1992; 165: $1082-1085$

${ }^{3}$ Hoover DR, Graham NMH, Murphy R, Vischer B, Anderson R, Mc Arthur J. An epidemiologic analysis of Mycobacterium avium complex disease in homosexual men infected with human immunodeficiency virus type 1 . Clin Infect Dis 1995; 20: 12501258

${ }^{4}$ Wallace RJ, Nash DR, Tsukamura M, Blacklock ZM, Silcox VA. Human Disease due to Mycobacterium smegmatis. J Infect Dis 1988; 158: $52-58$
${ }^{5}$ Wayne LG, Good RC, Krichevsky MI, Blacklock Z et al. Fourth report of the cooperative open-ended study of slowly growing mycobacteria of the International Working Group on Mycobacterial Taxonomy. Int J Syst Bacteriol 1991; 41: 463-472

${ }^{6}$ Lincoln EM, Gilbert LA. Disease in children due to mycobacteria other than Mycobacterium tuberculosis. Am Rev Respir Dis 1972; 158: $52-59$

${ }^{7}$ Cox EG, Heil SA, Kleiman MB. Lipoid Pneumonia and Mycobacterium Smegmatis. Ped Infect Dis J 1994; 13: 414- 416

${ }^{8}$ Pierre-Audigier C, Jouanguy E, Lamhamed S, Altare F, Rauzier J, Vincent V, Canioni D, Emile JF, Fischer A, Blanche S, Gaillard JL, Casanova JL. Fatal disseminated Mycobacterium smegmatis infection in a child with inherited interferon gamma receptor deficiency. Clin Infect Dis 1997; 24: $982-984$

${ }^{9}$ Von Moos S, Leuenberger PH, Beer V, DeHaller R. Infection pleuro-pulmonaire a Mycobacterium smegmatis. J Infect Dis 1986; 116: $1852-1856$

${ }^{10}$ Aronchick JM, Miller MT, Epstein DM, Gefter WB. Association of achalasia and pulmonary mycobacterium fortuitum infection. Radiology 1986; 86: 85-86

${ }^{11}$ Varghese G, Sheperd R, Watt P, Bruce HJ. Fatal infection with mycobacterium fortuitum associated with oesophageal achalasia. Thorax 1988; 43: $151-152$

12 Schulz KT, Fischer P. Koinzidenz von Lungenerkrankungen durch Mycobacterium fortuitum und Ösophagusachalasie. Kasuistik und Literaturübersicht. Pneumologie 1992; 46: 576-579

${ }^{13}$ Hadjiliadis D, Adlakha A, Prakash BS. Rapidly Growing Mycobacterial Lung Infection in Association With Esophageal Disorders. Mayo Clin Proc 1999; 74: 45-51

${ }^{14}$ Langdon DE. Mycobacterial lung infections. Mayo Clin Proc 1999; 74: 44-45

${ }^{15}$ Newton JA, Weiss PJ. Aspiration Pneumonia Caused by Mycobacterium smegmatis. Mayo Clin Proc 1994; 69: 296-298

${ }^{16}$ Konietzko N, Kassen U. Atypische Mykobakteriosen. AtemwLungenkrh 1990; 16: 109-115

${ }^{17}$ Petersen K. Durch atypische Mykobakterien verursachte Erkrankungen: Diagnose. Prax Klin Pneumol 1988; 42: 606-609

${ }^{18}$ Wolinsky E. Non-tuberculous mycobacteria and associated disease (State of the art). Am Rev Respir Dis 1979; 119: 107-159

${ }^{19}$ Greinert U, Schlaak M, Rüsch-Gerdes S, Flad H-D, Ernst M. Low In Vitro Production of Interferon- $\gamma$ and Tumor Necrosis Factor $\alpha$ in HIV-Seronegative Patients with Pulmonary Disease Caused by Nontuberculous Mycobacteria. J Clin Immunol 2000; 20: 445452

${ }^{20}$ Nathan CF, Murray HW, Wiebe ME, Rubin BY. Identification of interferon-gamma as the lymphokine that activates human macrophage oxidative metabolism and antimicrobial activity. J Exp Med 1983; 158: 670-689

${ }^{21}$ Schluger NW, Rom WN. The host immune response to tuberculosis. Am J Respir Crit Care Med 1998; 157: 679-691

22 Kindler V, Sappino AP, Grau GE, Piquet PF, Vassali P. The inducing role of tumor necrosis factor in the development of bacterial granulomas during BCG infection. Cell 1989; 56: 731 - 740

23 Parronchi P, DeCarli M, Manetti R, Simonelli C, Sampognaro S, Piccini MP, Macchia D, Maggi E, DelPrete G, Romagnani S. IL4 and IFN (alpha and gamma) exert opposite regulatory effects on the development of cytolytic potential by $\mathrm{TH} 1$ and $\mathrm{TH} 2$ cell clones. J Immunol 1992; 149: 2977 - 2983

${ }^{24}$ Wolf HM, Eibl MM, Georgi E, Samstag A, Spatz M, Uranus S, Passl R. Long-term decrease of CD4+CD45RA+ T cells and impaired primary immune response after post-traumatic splenectomy. $\mathrm{Br}$ J Haematol 1999; 107: 55-68

${ }^{25}$ Tsai MC, Lin SL, Chuang CY. Changes in T-lymphocyte subpopulations in patients splenectomized for trauma. J Formos Med Assoc 1991; 90: $240-243$ 
${ }^{26}$ Lanng Nielsen J, Haahr S. Interferon production, cellular and humoral immunity in splenectomized patients. Scand J Haematol 1982; 29: $168-174$

27 Diagnosis and Treatment of Disease Caused by Nontuberculous Mycobacteria. Statement of the American Thoracic Society. Am J Respir Crit Care Med 1997; 156: 1 - 25

${ }^{28}$ Miller WT. Spectrum of Pulmonary nontuberculous mycobacterial infection. Radiology 1994; 191: $343-350$

${ }^{29}$ Erasmus JJ, McAdams HP, Farrell MA, Patz EF. Pulmonary nontuberculous myco-bacterial infection: radiologic manifestations. Radiographics 1999; 19: 1487-1505

${ }^{30}$ Bailey WC. Treatment of atypical mycobacterial disease. Chest 1983; 84: 625-628

31 Schönfeld N, Schaberg T, Loddenkemper R. Neue Entwicklungen bei nicht-tuberkulösen Mykobakteriosen. Pneumologie 1996; 50: $313-322$

32 Urbanczik R. Probleme im Zusammenhang mit der Chemotherapie von Mykobakteriosen verursacht durch so genannte atypische Mykobakterien. Prax Klin Pneumol 1988; 42: 610-612
Dr. med. J. Schreiber

Städtisches Klinikum Dessau

Klinik für Innere Medizin

Abteilung für Pneumologie

06847 Dessau

E-mail: skd.innere2@t-online.de 